International Journal on Cybernetics \& Informatics (IJCI) Vol. 5, No. 4, August 2016

\title{
ANALYSIS OF INERTIAL SENSOR DATA USING TRAJECTORY RECOGNITION ALgORITHM
}

\author{
Nagadeepa.Ch. ${ }^{1}$, Dr.N.Balaji ${ }^{2}$ and Dr.V.Padmaja ${ }^{3}$ \\ ${ }^{1,3}$ Department of ECE, VNR-VJIET, Hyderabad, India. \\ ${ }^{2}$ Department of ECE, JNTU Vizianagaram, JNTUK, India.
}

\begin{abstract}
This paper describes a digital pen based on IMU sensor for gesture and handwritten digit gesture trajectory recognition applications. This project allows human and Pc interaction. Handwriting Recognition is mainly used for applications in the field of security and authentication. By using embedded pen the user can make hand gesture or write a digit and also an alphabetical character. The embedded pen contains an inertial sensor, microcontroller and a module having Zigbee wireless transmitter for creating handwriting and trajectories using gestures. The propound trajectory recognition algorithm constitute the sensing signal attainment, pre-processing techniques, feature origination, feature extraction, classification technique. The user hand motion is measured using the sensor and the sensing information is wirelessly imparted to PC for recognition. In this process initially excerpt the time domain and frequency domain features from pre-processed signal, later it performs linear discriminant analysis in order to represent features with reduced dimension. The dimensionally reduced features are processed with two classifiers State Vector Machine (SVM) and k-Nearest Neighbour (kNN). Through this algorithm with SVM classifier provides recognition rate is $98.5 \%$ and with $k N N$ classifier recognition rate is $95.5 \%$.
\end{abstract}

\section{KEYWORDS}

Trajectory recognition algorithm, Gesture trajectories, inertial sensor, Linear discriminant analysis, SVM, kNN Classifier.

\section{INTRODUCTION}

Gesture Recognition has become a rapid development search area and many techniques have been proposed to do the gesture trajectory recognition efficiently. Gesture recognition can be categorising into two: online and offline systems and these can be used for human wireless interaction with the computer.

Shengliet.al. [1] presented a Micro (IMU) based on (MEMS) sensor to sense the gesture motion information and mainly focused on human interaction with pc using characters and gesture recognition. Meenaakumariet.al. [2] presents pen with a MEMS accelerometer with three axes and a wireless zigbee transmission module for sensing, collecting and sending it to computer. Accelerations of hand gesture trajectories and display it on the computer. The drawback is displaying the gestures in seven segment display format. Renukaet.al. [3] processed the sensed data while it was under creation so this procedure falls under the category of online character recognition system. However, complexity increased because speed, pressure and strokes to generate a variety of motions differ for different users. Jeen-Shinget.al. [4] developed a portable pen-type device with accelerometer and RF transmission module and a trajectory recognition

DOI: 10.5121/ijci.2016.5412 
International Journal on Cybernetics \& Informatics (IJCI) Vol. 5, No. 4, August 2016

algorithm. Users can create hand gestures at comfortable level with ordinary velocity. Raw signal acquisition, signal preprocessing, obtaining features, selection of valid features, and feature extraction are the steps involved in trajectory recognition algorithm [5]-[12]. In the paper S.Zhang et.al acceleration signal detection, processing and recognition using Hidden Markov Model (HMM) have been performed. In the paper [13], PCA is applied on the features acceleration, speed and position to reduce the feature dimension size. Then HMM with DTW were applied to get the recognition accuracy is $90 \%$. Krishnan et al. proposed to calculate the frequency and time domain attributes of the accelerated signal simultaneously, by placing the sensor on various places on hand of the user. To classify hand motions three techniques named HMM, AdaBoost and k-NN classifier were used, in that AdaBoost classifier achieved an accuracy of $86 \%$.

Taking into consideration the entire literature survey this paper was an implementation of trajectory recognition using an inertial sensor (accelerometer and gyroscope), a microcontroller board, and a wireless zigbee transmission module which is embedded into a pen for sensing and data acquisition. The proposed algorithm used a process as sensing accession, pre processing of raw signals, feature generation, feature extraction and two classification techniques. The Trajectory recognition procedure initially extracts the features with respect to both frequency domain features and time domain features from the inertial sensor signals later performs a linear discriminant analysis to represent the features with reduced dimension set. The reduced features are sent to two classifiers -state vector machines and $\mathrm{kNN}$ for better recognition.

The contributions of the project can be pointed as follows:

1. Trajectory Recognition using matlab with two efficient classification techniques.

2. Comparison of types of SVM and $\mathrm{kNN}$ and deriving the conclusion of best classifier for better recognition of each Telugu alphabet.

\section{Proposed System}

The proposed system involves the collection of the experimental data from the given experimental setup and then sending the data wirelessly using Zigbee transmission to the laptop which consists of MATLAB software.

Here, an offline gesture recognition system has been introduced which includes the following components:

- Digital pen

- Laptop with Zigbee receiver

The digital pen senses the hand motions for each handwritten digit or alphabet using an inertial sensor 6 DOF which contains an accelerometer sensor -3axis and gyroscope sensor-3axis and the sensed data is converted in to digital using a microcontroller and transmits the data wirelessly via ZIGBEE technology. Then laptop receives mathematical array of values for each handwritten digit taken from the user via ZIGBEE receiver. The dataset was prepared from various users and is loaded into Matlab. Then analyse the gesture trajectories using two classification techniques and checked for its recognition accuracy. 


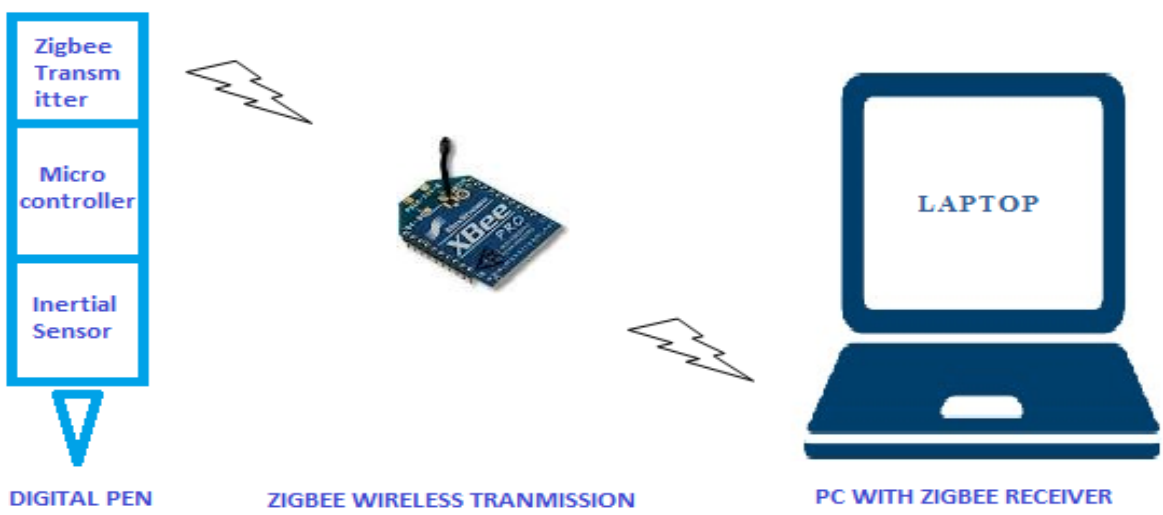

Figure1. Gesture Recognition System

\section{SYSTEM FLOW}

\subsection{Raw Data}

In this stage, the data corresponding to the different gestures are in the pattern of Telugu characters. These three gesture are considered for analysing our trajectory recognition algorithm and these patterns are collected from MPU6050. The database which is acquired consists of 300 samples for each letter per person built by attaining gestures from five persons.

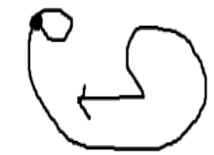

i) $/ \mathrm{a} /$

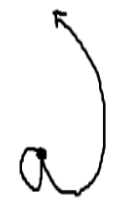

ii) /e/

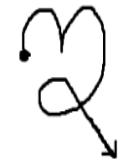

iii) /I/

Figure 2. Gestures written by various users

The figure 2 shows the gesture are in the pattern of Telugu alphabets taken from different users, these are the Telugu alphabets and their pronunciation is given as follows:
ii)
/a/
ii) /e/
iii) /I/

The gesture trajectory starts from '?' and ends at the 'arrow' symbol. All users perform the gestures without any or with minimal tilting of the sensor. We constrained the area to a square of $30 \mathrm{~cm} * 30 \mathrm{~cm}$ and at a height from the ground is $75 \mathrm{~cm}$. The transmitter and receiver are separated by a distance of $490 \mathrm{~cm}$. At each and every position of the sensor while writing it results out as 6 values in that first three values are gyroscope $\mathrm{x}-, \mathrm{y}-, \mathrm{z}-$ axis and other three values are accelerometer $\mathrm{x}-, \mathrm{y}-, \mathrm{z}-\mathrm{axis}$. After collecting the data from inertial sensor by the movement of hand for a single pattern obtain the data size of $300 * 6$ matrix in .txt format. The numerical data is 
stored in the templates folder with names of A, AE, EE ...etc. For training the system the data for each gesture is collected from eleven different people.

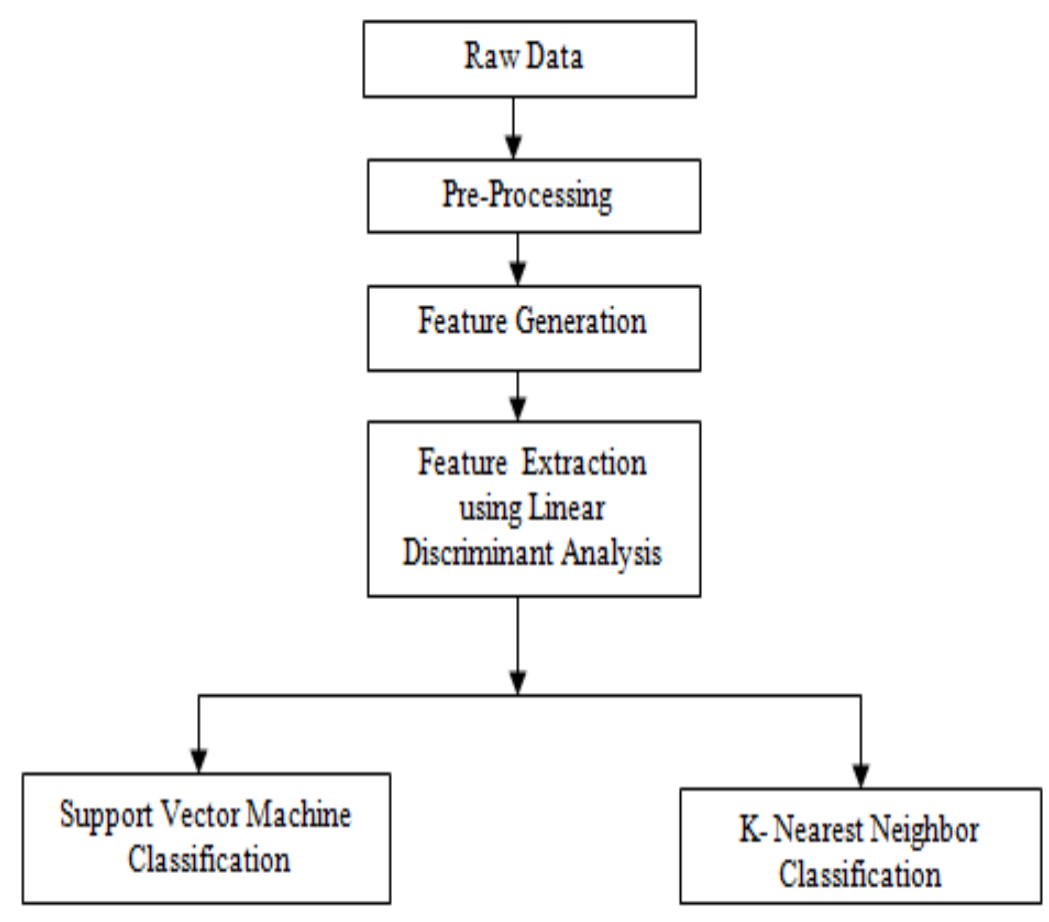

Figure 3. Hand Gesture Trajectory Recognition

The figure 3 illustrates step wise work flow of the entire gesture recognition system. The raw data matrix is initially loaded into matlab and it undergoes pre processing to discard high frequency noise and gravitational acceleration values. Then seven different features are generated and are represented in lower dimension using LDA for efficient classification using SVM and kNN techniques.

\subsection{Preprocessing}

\subsubsection{Moving Average Filter}

First, the obtained values undergo calibration for removal of drift errors and offsets. In signal pre processing stage utilizing moving average filter in order to reduce the high-frequency noise which present in the calibrated accelerations. Its functionality is, to produce a single output point by taking the average of $\mathrm{N}$ samples at a time. In this paper window size is eight for moving average filter.

\subsubsection{High Pass Filter}

A high-pass filter is utilised to eliminate the gravitational acceleration from the acceleration caused by hand movement. It is not applied on Gyroscope data. 


\subsection{Feature Generation}

The process of taking disorganised data and defining features are used in analytical study of given data. The gathered features are more informative and non-Redundant when compared to raw data. Here, seven features are generated: Mean, standard deviation, variance, Inter quartile range, correlation, Mean absolute deviation, RMS.

After all the features have been generated in feature extraction that's a special style of dimensionality reduction is applied to the info to get the foremost relevant data from the initial data and represent that information during a lower dimensionality area. It starts from an associate initial set of measured information and select the derived features meant to be more informative and non-redundant, leads to subsequent learning and generalization steps, and also resulting in higher human interpretations. There are many techniques available but in this Linear Discriminant Analysis is employed.

\subsection{Classification}

To the obtained dimensionality reduced feature vectors different classification techniques have been applied to classify the data into different classes. Comparison tables have been drawn for different classification accuracies have been drawn in the results below.

\section{RESULTS}

The figure 4 shows exporting the inertial sensor data of Telugu alphabet "@ ".

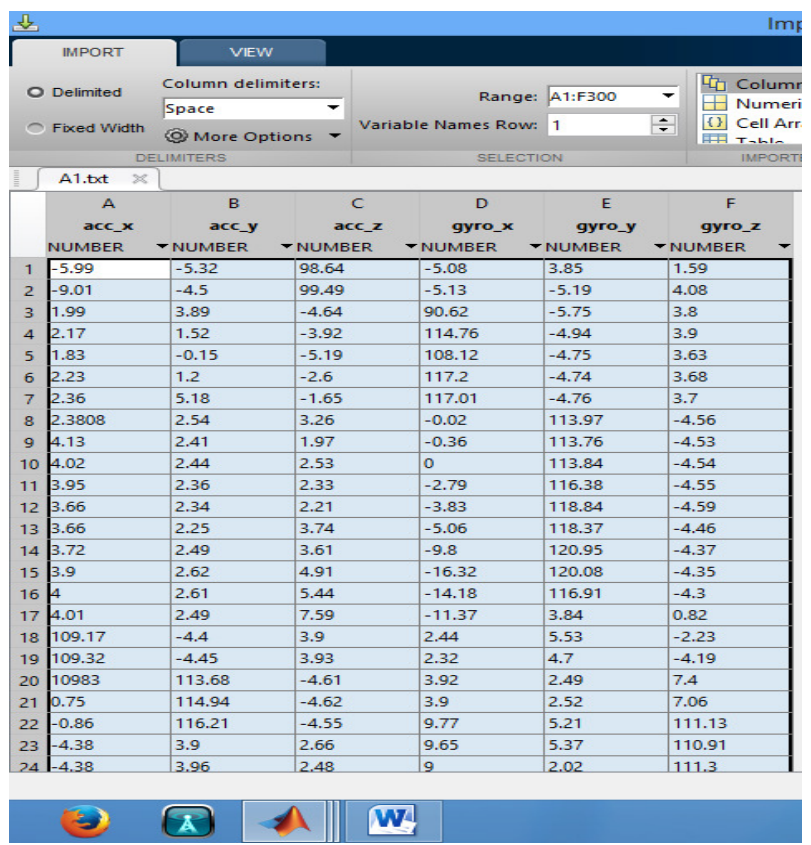

Figure 4. Importing data array into Matlab

The saved text file from the PC can be imported into matlab using the shortcut import button on the matlab start window. Each column represents inertial sensors axis data: 
International Journal on Cybernetics \& Informatics (IJCI) Vol. 5, No. 4, August 2016

Column1: acc_x

Column4: gyro_x

Column2: acc_y

Column5: gyro_y

Column3: acc_z

Column6: gyro_z
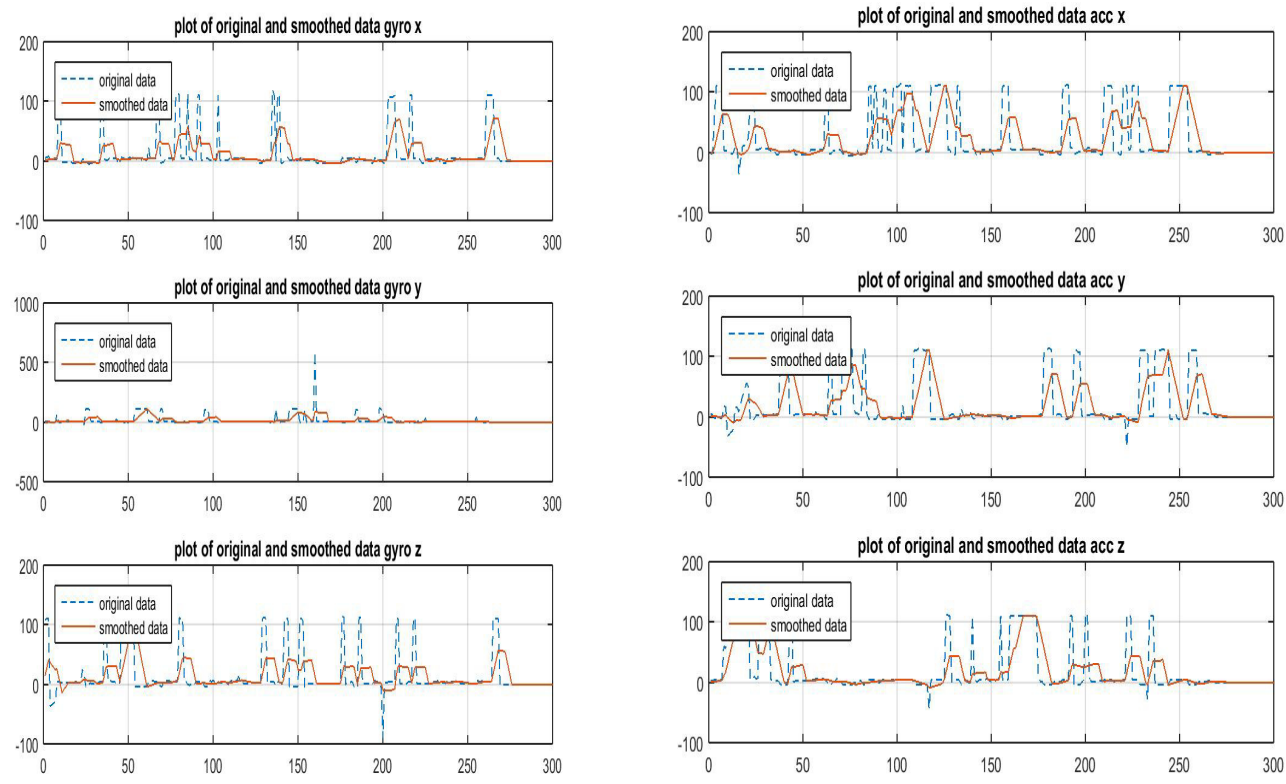

Figure 5. Gyro axis ouputs comparison before and after MVG filter

The figure 5 shows the graphically plotted gyroscopic sensor and accelerometer sensor each axis raw data compared with the moving average filtered data. Moving Average (MVG) Filter has been applied to each column in the obtained array and its functionality is to removes high frequency noise from the raw data.
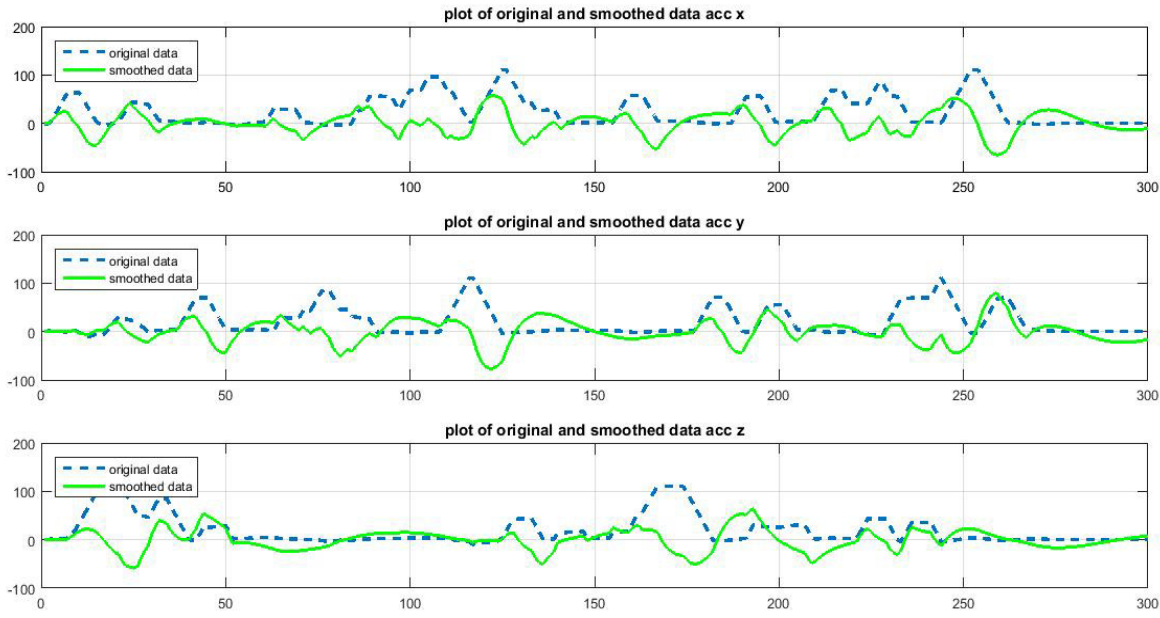

Figure 6. High pass filter output applied for only accelerometer data 
The MVG filtered accelerometer data is passed through a High pass filter which removes gravitational acceleration. Above figure shows the comparison graphs of before and after filtering through high pass filter.

After the steps of preprocessing, Feature generation and extraction the Feature columns of eleven writers of each alphabet are concatenated and are taken as predictors and the classes of column is taken as response.

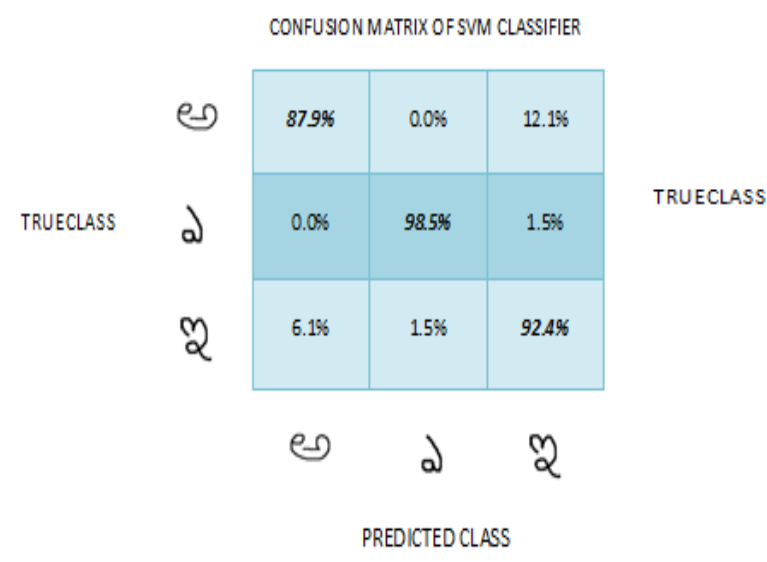

(a)

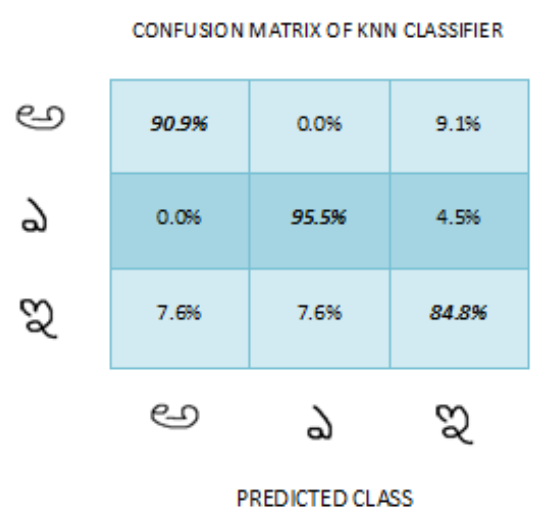

(b)

Figure 7. Confusion matrix with (a) SVM classifier and $\quad$ (b) KNN classifier

Figure 7 shows the confusion matrix of each classifier which indiactes the percentage of correct classification of each gesture pattern. The classification of SVM for gesture /e/ is $98.5 \%$ and it is more when compared with $\mathrm{KNN}$ for the same gesture is $95.5 \%$.

\section{CONCLUSIONS}

This paper proposed Telugu alphabet recognition system entirely relying on data from a 3-axis accelerometer and gyroscope. This paper has offered an efficient algorithm skeleton which can build constructive classifier for sensor based gesture recognition and handwriting. The proposed framework comprises hand motion acquisition, pre-processing of the sensing signal, feature generation and extraction, classification technique. With the decreased features, an SVM and KNN can be trained as powerful classifier. In this, we make use of two dimensional hand written Telugu character pattern gestures to validate the efficacy of the algorithm. The gesture recognition rate for SVM is $98.5 \%$ as well as for $\mathrm{KNN}$ is $95.5 \%$. This end result empowers that the likelihood of usage of embedded pen as a powerful tool chain for computer human interaction applications. 
International Journal on Cybernetics \& Informatics (IJCI) Vol. 5, No. 4, August 2016

\section{REFERENCES}

[1] S.Wang,Y.L.Hsu,J.N.Liu,“A measurement unit based pen with a trajectory reconstruction Algorithm and its applications,” IEEE Trans. Ind. Electron., vol. 57, no. 10, pp. 3508-3521, Oct 2010.

[2] Shengli Zhou1, Wen J. Li1, Zhuxin Dong1, Chung Ping Kwong "Hand-Written Character Recognition Using MEMS Motion Sensing Technology," Proceedings of the 2008 IEEE/ASME International Conference on Advanced Intelligent Mechatronics 2nd - 5th July 2008.

[3] M.Muthulakshmi, Meenaakumari.M, "MEMS Accelerometer based Hand Gesture Recognition", International Journal of Advanced Research in Computer Engineering \& Technology (IJARCET) vol. 2, No 5, pp. 1866-1892, May 2013.

[4] Renuka R, Suganya V, Arunkumar.B, "Online Hand Written Character Recognition Using Digital Pen For Static Authentication”, Proceedings of IRAJ International Conference, 20th October 2013, Chennai, India. ISBN: 97893-82702-34-4.

[5] L. Bao, S.S.Intille, "Activity recognition from user-annotated acceleration data," Pervasive, Lecture Notes in Computer Science, no. 3001, pp. 1-17, 2004.

[6] J.Y.Yang, Y.P.Chen, S. N. Liou, J. S. Wang, G.Y.Lee, "Online classifier construction algorithm for human activity detection using a tri- axial accelerometer," Appl. Math. Comput. pp. 849-860, Nov. 2008.

[7] Jeen-Shing Wang, Fang-Chen Chuang, "An Accelerometer-Based Digital Pen with a Trajectory Recognition Algorithm for Handwritten Digit and Gesture Recognition,” IEEE Sens. J., vol. 59, no. 07, pp. 1543-1551, July 2012.

[8] S. J. Preece, J. Y. Goulermas, L. P. J. Kenney, D. Howard, “A comparison of feature extraction methods for the classification of dynamic activities from accelerometer data," IEEE Trans. Biomed. Eng., vol. 56, no. 3, pp. 871879, Mar. 2009

[9] E. Sato, F. Harashima,T. Yamaguchi, "Natural interface using pointing behaviour for human-robot gestural interaction," IEEE Transactions Ind. Electron., vol. 54, no. 2, pp. 1105-1112, April 2007.

[10] Z. Dong, U. C. Wejinya, W. J. Li, "An optical-tracking calibration method for MEMS-based digital writing instrument,” IEEE Sensor Journal, vol. 10, no. 10, pp. 1543-1551, Oct. 2010.

[11] A. D. Cheok, Y. Qiu, K. Xu, K. G. Kumar, "Combined wireless hard- ware and real-time computer vision interface for tangible mixed reality," IEEE Trans. Ind. Electron., vol. 54, no. 4, pp. 2174-2189, Aug. 2007.

[12] Y. S. Kim, B. S. Soh, and S.-G. Lee, “A new wearable input device: SCURRY,” IEEE Trans. Ind. Electron., vol. 52, no. 6, pp. 1490-1499, Dec. 2005.

[13] S. D. Choi, A. S. Lee, S. Y. Lee, "On-line handwritten character recognition with 3D accelerometer," in Proc. IEEE Int. Conf. Inf. Acquisition, 2006, pp. 845-850.

[14] N. C. Krishnan, C. Juillard, D. Colbry, and S. Panchanathan, "Recognition of hand movements using wearable accelerometers,” J. Ambient Intell. Smart Environ vol. 1, no. 2, pp. 143-155, Apr. 2009.

\section{AUTHORS}

NagaDeepa.Ch received the B.Tech. Degree in Electronics \& Communications and the M.tech degree in embedded systems from the Jawaharlal Nehru Technological University of Hyderabad, India. She is currently pursuing the Ph.D. and working as assistant professor in the Department of ECE,VNRVJIET. Her main research interests include signal processing and pattern recognition techniques applied to electronic mobile devices.

Dr.N.Balaji obtained his B.E. degree from Andhra University. He received Master's and Ph.D degree from OsmaniaUniversity, Hyderabad. Presently he is working as a professor in the Department of ECE, JNTUK University college of Engineering Vizianagaram, Andhra Pradesh. He has authored more than 40 Research papers in National and International Conferences and Journals. He is a life Member of ISTE and Member of VLSI Society of India. His areas of research interest are VLSI, Signal Processing, Radar.

Dr.V.Padmaja born in 1968. She received B.E. Degree in Electronics and Communications Engineering, M.E Degree in Digital Systems Engineering from O.U in 1991 and 1999 respectively. She received Ph.D from J.N.T.U in 2009. She is an Professor in Dept. of ECE in VNRVJIET, her research of interest includes image processing and Embedded Systems. She has authored more than 6 Research papers in National and International Conferences and Journals.
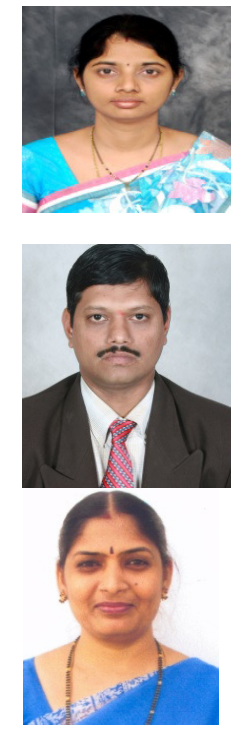